\title{
Sounding and Signal Simulation of Complex Surface and Subsurface Structures for the WISDOM GPR
}

Christoph Statz et al. '

Key in the interpretation and understanding of WISDOMs ground penetrating RADAR (GPR) measurements is the capability to correctly (and efficiently) simulate the instrument characteristics and the RADAR wave propagation in the Martian subsurface (the signal received by WISDOM), taking into account all relevant effects at large scale. In this contribution we present a ray tracing approach that can be applied to heterogeneous and inhomogeneous media and includes the antenna characteristics of the WISDOM instrument as well as rover structures.

The WISDOM GPR is part of the 2022 ESA-Roscosmos ExoMars "Rosalind Franklin" rover payload. It will probe the Martian surface and subface at centimetric resolution and a penetration depth of about $3 \mathrm{~m}$. WISDOMs primary scientific objective is the high-resolution characterization of the material distribution within the first few meters of the Martian subsurface as a contribution to the search for evidence of past life [1] and to support the drilling operations [2].

The simulation tool consists of two parts: The first part simulates the instrument at system level and generates the signal that is fed into the antenna as well as the receive-filter and discretization characteristic of the instrument (taking into account filters, RF effects and the ADC). The second part simulates the wave propagation of this signal in complex media (inhomogeneous or heterogeneous lossy media) taking into account polarization effects and the WISDOM antenna pattern [3]. This method is a hybrid between conventional raytracing (SBR), differential raytracing and physical optics. The simulation complexity can be granularly controlled and weighed against the level of approximation. It is capable of simulating electrically large domains with an acceptable accuracy yielding good predictions of the propagation properties in Martial soil while being significantly less computationally expensive than conventional full-wave solvers like FEM or the Finite-Differences in TimeDomain Method.

The results of the system-level-simulation and the propagation simulation for multiple measurement positions (along a rover track) are then combined (similar to the application of a filter) in order to generate a synthetic radargram. This radargram can be directly compared to the WISDOM measurements.

The proposed method is validated using measurements of the WISDOM instrument at analog sites and by reference simulations using the FDTD Method [4]. We present synthetic radargrams as simulation results for several sounding scenarios including the WISDOM antenna characteristics, an inhomogeneous subsurface and lossy materials.

The proposed approximation method yields accurate estimates of WISDOM soundings for a complex subsurface while being significantly faster than conventional (full wave) methods. The synthetic radargrams can easily be compared to actual measured data.

The research on WISDOM is supported by funding from the Centre National d'Etudes Spatiales (CNES) and the Deutsches Zentrum für Luft- und Raumfahrt (DLR).

[1] V. Ciarletti, C. Corbel, D. Plettemeier, P. Cais, S. M. Clifford, S.-E. Hamran, "WISDOM GPR Designed for Shallow and High-Resolution Sounding of the Martian Subsurface", Proceedings of the IEEE, Vol. 99, Issue 5, pp. 824-836, May 2011.

[2] V. Ciarletti, S. Clifford, D. Plettemeier and the WISDOM Team, "The WISDOM Radar: Unveiling the Sub surface Beneath the ExoMars Rover and Identifying the Best Locations for Drilling", Astrobiology, Vol. 17, No. 6-7, July 2017

[3] D. Plettemeier et al., "Full polarimetric GPR antenna system aboard the ExoMars rover," 2009 IEEE Radar Conference, Pasadena, CA, 2009, pp. 1-6, doi: 10.1109/RADAR.2009.4977120.

[4] C. Statz and D. Plettemeier, "BETSi: An electromagnetic time-domain simulation tool for antennas and heterogeneous media in ground penetration radar and biomedical applications," 2017 Computing and Electromagnetics International Workshop (CEM), Barcelona, 2017, pp. 3738, doi: $10.1109 /$ CEM.2017.7991875.

How to cite: Statz, C., Plettemeier, D., Lu, Y., Benedix, W.-S., Hegler, S., Ciarletti, V., Le Gall, A., Corbel, C., Oudart, N., and Hamran, S.-E.: Sounding and Signal Simulation of Complex Surface and Subsurface Structures for the WISDOM GPR, Europlanet Science Congress 2020, online, 21 September-9 Oct 2020, EPSC2020-663, https://doi.org/10.5194/epsc2020-663, 2020 
Comments on the presentation

Login to access the discussion

\section{Contact}

Imprint

Contact 